\title{
Stimulus-outcome learnability differentially activates anterior cingulate and hippocampus at feedback processing
}

\author{
Paul F. Rodriguez ${ }^{1}$ \\ Department of Radiology, University of California, San Diego, La Jolla, California 92037, USA
}

\begin{abstract}
Memory systems are known to be influenced by feedback and error processing, but it is not well known what aspects of outcome contingencies are related to different memory systems. Here we use the Rescorla-Wagner model to estimate prediction errors in an fMRI study of stimulus-outcome association learning. The conditional probabilities of outcomes for a given stimulus are manipulated so that associations are either learnable or unlearnable (pseudorandom). The delay between stimulus and outcome is jittered so that we can separately compare activity for either stimulus processing or feedback processing. We find that hippocampus and anterior cingulate are differentially active primarily at feedback processing: Learnable associations are correlated with significantly more hippocampal activity and significantly less anterior cingulate activity than unlearnable associations. We also find that positive prediction errors modulate feedback processing in the midbrain for both types of associations. We suggest that learnable associations use more declarative memory, unlearnable associations involve more uncertainty monitoring, and, in both kinds of associations, positive prediction errors provide a reinforcement signal.
\end{abstract}

[Supplemental material is available online at www.learnmem.org.]

There is substantial neuropsychological evidence for the existence of multiple memory systems, which are functionally and neurally distinct (Cohen and Squire 1980; Squire 1992; Eichenbaum et al. 1994; Gabrieli et al. 1997; Packard and Knowlton 2002; White and McDonald 2002). The striatum, which supports a number of forms of skill learning and stimuli-response learning, is implicated in procedural memory. The medial temporal lobe (MTL), which supports flexible representations, novel associations, and episodic memory, is implicated in declarative memory.

Despite the evidence for the procedural/declarative distinction, it is not well understood what factors will bias learning and memory toward one system or the other, when either system could be involved in a task. In the case of associative learning, there is evidence that learning strategies are important. Poldrack et al. (2001) found that paired-observation learning, in which subjects observe a stimulus and an outcome and execute the motor response to select that outcome, will result in more hippocampal activity. In contrast, trial-and-error learning, in which subjects make predictions and the outcome is monitored as feedback, will produce more activity in the striatum (see also Cincotta and Seger 2007).

In this work, we propose that another factor for memory system involvement is the learnability of stimulus-outcome (S-O) associations. Learnable associations, as opposed to unlearnable associations, have outcomes that are predictable greater than chance. Consequently, over time subjects will experience positive feedback more often for learnable associations and negative feedback more often for unlearnable associations. We hypothesize that because of these feedback experiences, subjects will recruit alternative learning strategies, and hence different brain regions, for the two types of S-O associations. However, it is not obvious if those differences will be reflected in neural activity for stimulus processing, feedback processing, or both. If the controlling factor

\footnotetext{
'Corresponding author.
}

E-mail p4rodrig@ucsd.edu; fax (858) 534-1078.

Article is online at http://www.learnmem.org/cgi/doi/10.1101//m.1191609. for memory system activation depends on monitoring outcomes, then one might expect differences in feedback processing regions, such as the anterior cingulate cortex (ACC), because the unlearnable associations have high error rates. Alternatively, if the strength of outcome predictions is a controlling factor, then one might expect differences in stimulus processing regions, such as the MTL, because learnable associations will produce stronger, more confident predictions.

The effect of positive or negative feedback can also be represented by the prediction error. Many fMRI studies have used mathematical models of reward learning to show that prediction errors correlate with activity in dopaminergic target regions, as suggested by neurophysiologic data (for review, see Schultz 2002; Montague et al. 2004). Recently, we have also shown in a nonrewarded probabilistic classification task called "weather prediction" that prediction errors correlate with ventral striatal fMRI activation (Rodriguez et al. 2006). Prediction errors were modeled with a variant of the Rescorla-Wagner model (Rescorla and Wagner 1972), which has been used effectively in human category learning (Gluck and Bower 1988) and has been applied to other fMRI analyses of nonreward associative learning (Fletcher et al. 2001; Glascher and Buchel 2006; Yacubian et al. 2006).

In this work, we use the Rescorla-Wagner model to estimate prediction errors for both unlearnable and learnable S-O associations. The task is a simplified version of the weather prediction task (Knowlton et al. 1996), which consistently shows evidence of procedural learning with increased activation in frontal-striatal circuits (Poldrack et al. 2001; Beninger et al. 2003; Aron et al. 2004; Moody et al. 2004; Shohamy et al. 2004; Foerde et al. 2006). In the version here, one of four complex, unfamiliar stimuli are associated with three possible outcomes. Two stimuli have strong S-O associations that are relatively easy to learn, and the other two stimuli have pseudorandom $\mathrm{S}-\mathrm{O}$ associations that are essentially unlearnable in the sense that outcomes are unpredictable.

The main comparison of interest is between the learnable and unlearnable conditions, at the time of either stimulus or feedback processing, with and without prediction error modulation. The 
main result is that at feedback processing there is a cluster in the hippocampus of greater activity for learnable S-O associations, and there is a cluster in the ACC of greater activity for unlearnable $\mathrm{S}-\mathrm{O}$ associations. There is also modulation of midbrain activity by positive prediction errors for both learnable and unlearnable S-O associations, again at feedback processing. We conclude that learnability has some effect on memory systems during feedback processing, and discuss the possible interpretations with respect to declarative memory and error monitoring functions.

\section{Results}

\section{Behavioral results}

Fourteen subjects were trained on 144 trials while being scanned. The scanning was broken up into four scanning runs where each run consisted of nine trials for each stimulus (see Materials and Methods). All subjects acquired the learnable $\mathrm{S}-\mathrm{O}$ associations significantly above chance (mean percentage of correct $=70, t_{(13)}=$ 2.99, $P \sim 0.01$ ) (lower line in Fig. 1A). The increase from run 1 to run 2 was significant (paired $t$-test, $t_{(13)}=2.84, P<0.015$ ). There was also a significant drop in performance from run 2 to run 3 (paired $t$-test, $t_{(13)}=2.23, P \sim 0.044$ ), which reflects the fact that outcome probabilities were slightly changed in order to produce more trials with negative feedback (i.e., the fraction of trials for the most likely outcome for learnable stimuli changed from eight out of nine to seven out of nine; see Materials and Methods). The upper line in Figure 1A shows the mean percentage of trials in which subjects chose the most likely outcome (i.e., optimal prediction, overall mean 83.9\%). Again the increase from run 1 to run 2 was significant (paired $t$-test, $t_{(13)}=2.35, P \sim 0.0036$ ), and there was little change in the average of optimal predictions in the last two runs.

Individually, subjects vary in the amount of optimally choosing the most likely outcome versus nonoptimal behaviors, such as probability matching (see also Shanks et al. 2002). In the last two scanning runs, two subjects chose the most likely outcome in $100 \%$ of trials, two subjects chose it in at least $94.4 \%$ of trials, four subjects chose it in $91.6 \%$ of trials, and the other six chose it in a range of $66.6 \%-88.9 \%$ of trials. In other words, two subjects may have learned an optimal rule for their response, but all other subjects learned to choose the most likely outcome with a high probability.

As described in the Materials and Methods section, the percentage of positive feedback (i.e., where the outcome matches the prediction) for the unlearnable stimuli were fixed at chance level and are not shown.

There are two measurable behavioral changes after negative feedback that also indicates learning - the percentage of time subjects switched choices and the response time on the next trial.
A.

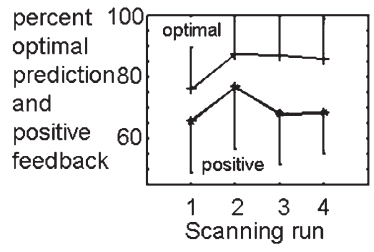

B.

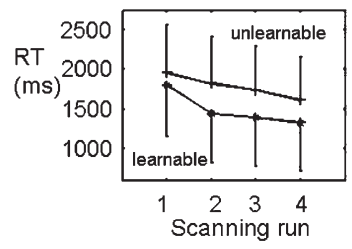

Figure 1. Subjects acquire the learnable associations by the second scanning run. (A) Percentage of correctly matching outcomes (i.e., positive feedback) and percentage of optimal predictions for learnable stimuli across the four scanning runs. (B) The average response time for learnable and unlearnable stimuli, relative to stimuli onset. The overall mean was about $1781 \mathrm{msec}$ for unlearnable stimuli and $1488 \mathrm{msec}$ for learnable stimuli.
Across the last two runs, after learning had stabilized, subjects switched their prediction an average of $49.2 \%$ after unlearnablestimulus negative feedback, and switched $34.6 \%$ after learnablestimulus negative feedback. In a paired $t$-test across subjects, the difference in percentage switching was significant $\left(t_{(13)}=2.80, P \sim\right.$ $0.015)$, meaning that subjects were switching choices significantly more often for unlearnable stimuli. Also, in the last two runs, the response time after negative feedback for learnable stimuli was significantly greater than after positive feedback (mean difference $123 \mathrm{msec}$, paired $t$-test, $t_{(13)}=2.52, P \sim 0.0256$ ), whereas for unlearnable stimuli, the difference was nearly zero (mean difference $3 \mathrm{msec}$, paired $t$-test $t_{(13)}=0.68, P \sim 0.51$ ).

The mean response time, as measured from the onset of the stimulus, across subjects for both learnable stimuli ( $\sim 1488 \mathrm{msec})$ and unlearnable stimuli $(\sim 1781 \mathrm{msec})$ decreased across scanning runs (Fig. 1B). The response time for learnable stimuli was significantly less than unlearnable stimuli in each run (paired $t$-test $P$-values for runs 1 to 4 , respectively, were as follows: $t_{(13)}=$ $2.25, P \sim 0.021 ; t_{(13)}=4.29, P<0.001 ; t_{(13)}=5.47, P<0.001 ; t_{(13)}=$ 5.94, $P<0.001$ ). The decrease in response time for unlearnable stimuli was possibly due to task familiarity, given that there was no particular S-O association to be learned. It is also possible that subjects learned that outcomes are unpredictable, so they made faster guesses. That may be true to some degree; however, it does not fully explain response behavior. In particular, for unlearnable stimuli, all subjects switched choices more often after negative feedback than after positive feedback in the last two runs (49\%$24 \%$, paired $t$-test, $\left.t_{(13)}=4.02, P \sim 0.0015\right)$. Thus, it seems that subjects were monitoring feedback to some extent, and not just making random guesses.

Immediately after the scanning ended, all subjects were asked to describe their knowledge of S-O associations and their main learning strategy. All subjects informally reported that some $\mathrm{S}-\mathrm{O}$ associations were easier to learn. Only two subjects thought that the probabilities had changed at the end. Eight out of 14 subjects claimed to label parts of the figure of learnable stimuli according to expected outcome (e.g., a circle would correspond to a sun or an oval to a rain drop); the other subjects claimed to be mostly guessing as their main strategy. Of course, this does not preclude that those who were guessing on some trials also assigned verbal labels for stimuli in other trials, and vice versa.

\section{FMRI results}

\section{Task versus baseline}

In a whole brain analysis of task versus baseline (i.e., a combination of all stimulus/response and feedback regressors in the design matrix), there was significant increased activation in occipital, parietal, and cerebellum regions (Table 1). In regions of interests (ROI) analysis, there was increased activation in putamen (Table 1). Activation in these regions has also been found in related studies (e.g., Poldrack et al. 2001; Aron et al. 2004; Seger and Cincotta 2005) and possibly reflect visual and motor processing aspects of the task.

In a whole brain analysis, there was decreased activation in ACC near the genu of the corpus callosum, post-central gyrus, and temporal pole (Table 1). No clusters of decreased activation were found in ROI analysis.

\section{Stimulus processing}

In a whole brain comparison of stimulus processing for unlearnable-learnable S-O associations, there were significant clusters of activity in left middle occipital gyrus $(-27,-78,24)$ and left inferior frontal lobe $(-24,3,36 ;-36,18,21$; Table 2$)$. There was nothing significant for the reverse comparison. 
Table 1. Contrast of effect sizes for all stimuli and feedback presentations versus baseline

\begin{tabular}{lcrrr}
\hline & P-value & $\begin{array}{c}\text { Cluster } \\
\text { size }\end{array}$ & $\begin{array}{c}t \text {-Value/ } \\
\text { Z-score }\end{array}$ & $\begin{array}{c}\text { MNI } \\
\text { coordinates }\end{array}$ \\
\hline Stimuli and feedback & baseline & & & \\
Occipital & $<0.001$ & 346 & $9.31 / 4.81$ & $-15,-96,6$ \\
& $<0.001$ & 102 & $7.60 / 4.62$ & $39,-84,18$ \\
Parietal & $<0.001$ & 65 & $6.04 / 4.10$ & $36,-51,48$ \\
Cerebellum & $<0.001$ & 104 & $5.47 / 3.87$ & $33,-63,-24$ \\
Occipito temporal & 0.015 & 34 & $4.75 / 3.56$ & $-48,-69,0$ \\
L Putamen & 0.027 (SVC) & 21 & $4.00 / 3.17$ & $-21,9,3$ \\
Stimuli and feedback & baseline & & & \\
ACC & $<0.001$ & 122 & $9.57 / 5.13$ & $0,36,6$ \\
Post-central gyrus & 0.005 & 41 & $7.29 / 4.52$ & $30,-48,63$ \\
Temporal pole & 0.003 & 44 & $6.52 / 4.27$ & $57,3,-9$ \\
\hline
\end{tabular}

For both learnable stimuli versus baseline and unlearnable stimuli versus baseline, there were significant clusters of increased activity in the posterior hippocampus (see Table 2). Although there was more activity in this region for unlearnable stimuli, the difference between them was not significant $(P \sim 0.140$ small volume corrections [SVC], at $24,-24,-9)$. In other ROI comparisons, there were no significant differences.

\section{Feedback processing}

As detailed in the Materials and Methods section, the conditions at feedback processing comprise a $2 \times 2$ design of learnability $\times$ valence. The contrasts will compare learnability, either within or collapsed across valence, and vise versa. Also, in order to remove possible confounds of unintended responses, the regressor for feedback processing after learnable stimuli only includes trials in which subjects correctly chose the most likely outcome (see Materials and Methods).

In a whole brain analysis of feedback processing after learnable stimuli versus feedback processing after unlearnable stimuli, there were no significant clusters of activity, either within or collapsed across positive and negative feedback.

In ROI analysis, there was significantly more activation in the hippocampus $(27,-15,-12)$ for feedback processing after learnable stimuli versus feedback processing after unlearnable stimuli, collapsed across valence. This focus is consistent, with data showing that the anterior portion of the hippocampus is important for learning associations and relations (Kirwan and Stark 2004; Binder et al. 2005; Davachi 2006). The plot of effect sizes shows that much of this difference is due to a decrease in activity for both positive and negative feedback processing after unlearnable stimuli (Table 3; Fig. 2A), with a slight increase for feedback processing after learnable stimuli.

Additionally, there was more activation in $\operatorname{ACC}(6,33,45)$ for feedback processing after unlearnable stimuli than after learnable stimuli, also collapsed across valence. That focus is located in the rostral cingulate zone, near the estimated boundary of the presupplementary motor area (see Picard and Strick 2001; Rushworth et al. 2004), which is thought to be involved in response conflict monitoring and behavioral adjustments (e.g., Botvinick et al. 2004; Kerns et al. 2004). The plot of effect sizes shows that much of the difference is to due an increase for unlearnable stimuli processing (see Table 3; Fig. 2B) for both positive and negative feedback.

In a whole brain comparison between positive and negative feedback, there was a cluster of increased activity in the superior parietal lobe for positive - negative feedback after learnable stimuli (see Table 3). No other whole brain or ROI comparison between positive and negative feedback processing produced significant clusters, within or collapsed across learnability.
In ROI analysis relative to baseline, we did find increased activity in the striatum for negative feedback after unlearnable stimuli. Although one might have expected differential activity in the striatum based on reward learning (e.g., Abler et al. 2006), one must bear in mind that feedback in this experiment is not a primary reward but information that the subject has to match up to his/her prediction.

\section{Parametric modulation with prediction error}

Figure 2C shows an example of positive and negative prediction errors for one subject derived from an implementation of the Rescorla-Wagner model (see Materials and Methods). The error values were entered in the statistical analysis as modulations of activity during feedback processing. In a whole brain analysis of modulation of feedback processing by positive prediction errors for learnable stimuli, there are significant clusters in right inferior frontal gyrus (IFG), cerebellum, and left posterior parietal compared to baseline (Table 4). This means there is higher activation for larger positive prediction errors. These are possibly related to general learning mechanisms and not motor movements per se because subjects typically make a response in less than $2 \mathrm{sec}$ and feedback is presented a minimum of $4.5 \mathrm{sec}$ after the onset of the stimulus (see Materials and Methods; Fig. 1). Other association studies have also found activation in frontal gyrus and/or cerebellum related to the amount of uncertainty or error (e.g., Berns et al. 2001; O'Doherty et al. 2003; Volz et al. 2003; Haruno et al. 2004).

In ROI analysis of modulation of feedback processing by positive prediction errors after learnable stimuli, there was a significant cluster in the midbrain compared with baseline $(0,-21$, 18; Table 4; Fig. 2C). Although it is difficult to identify midbrain regions at the spatial resolution of fMRI, this cluster is clearly in the region of substantia nigra and ventral tegmental area according to the atlas of Lucerna et al. (2002).

In ROI analysis of unlearnable stimuli, there was also a marginal trend in the midbrain $(P \sim 0.06$ SVC; $-9,-21,-12$; Table 4$)$ for modulation of feedback processing by positive prediction errors. Although not significant, the cluster is only a few millimeters away from one of the peaks for learnable-stimulus positive prediction errors.

No significant differences were found for modulation by negative prediction errors versus baseline for either learnable or unlearnable stimuli. And no significant differences were found for comparisons of modulation by positive and/or negative prediction errors between learnable and unlearnable stimuli.

\section{Discussion}

In summary, hippocampus and ACC were differentially activated during feedback processing, depending on learnability. Feedback processing for learnable associations involved relatively more

Table 2. Contrast of effect sizes for learnable and unlearnable stimuli processing

\begin{tabular}{|c|c|c|c|c|}
\hline & $P$-value & $\begin{array}{l}\text { Cluster } \\
\text { size }\end{array}$ & $\begin{array}{l}t \text {-Value/ } \\
Z \text {-score }\end{array}$ & $\begin{array}{c}\text { MNI } \\
\text { coordinates }\end{array}$ \\
\hline \multicolumn{5}{|c|}{ Unlearnable stimuli > learnable stimuli } \\
\hline Mid occipital gyrus & 0.020 & 74 & $4.90 / 3.62$ & $-27,-78,24$ \\
\hline Inf frontal gyrus & 0.002 & 106 & $4.87 / 3.61$ & $-36,18,21$ \\
\hline Precentral gyrus & 0.010 & 84 & $4.34 / 3.35$ & $-51,6,27$ \\
\hline \multicolumn{5}{|c|}{ Unlearnable stimuli > baseline } \\
\hline Hippocampus & 0.001 & 60 & $6.53 / 4.27$ & $24,-27,-9$ \\
\hline & 0.004 & 41 & $5.88 / 4.04$ & $-18,-33,-3$ \\
\hline \multicolumn{5}{|c|}{ Learnable stimuli > baseline } \\
\hline Hippocampus & .014 (SVC) & 24 & $5.22 / 3.77$ & $-24,-33,0$ \\
\hline
\end{tabular}


Table 3. Contrast of effect sizes for random-stimuli and learnable-stimuli feedback processing

\begin{tabular}{|c|c|c|c|c|}
\hline & $P$-value & $\begin{array}{l}\text { Cluster } \\
\text { size }\end{array}$ & $\begin{array}{l}t \text {-Value/ } \\
\text { Z-score }\end{array}$ & $\begin{array}{c}\text { MNI } \\
\text { coordinates }\end{array}$ \\
\hline \multicolumn{5}{|c|}{ Learnable S-O feedback > unlearnable S-O feedback } \\
\hline Hippocampus & 0.008 (SVC) & 33 & $4.67 / 3.51$ & $27,-15,-12$ \\
\hline \multicolumn{5}{|c|}{ Unlearnable S-O feedback > learnable S-O feedback } \\
\hline ACC/SMA & 0.029 (SVC) & 24 & $4.16 / 3.26$ & $6,33,45$ \\
\hline \multicolumn{5}{|c|}{ Unlearnable $\mathrm{S}-\mathrm{O}$ negative feedback > baseline } \\
\hline Left striatum & 0.025 (SVC) & 20 & $4.46 / 3.41$ & $-18,9,3$ \\
\hline Right striatum & 0.028 (SVC) & 19 & $4.51 / 3.44$ & $15,18,9$ \\
\hline \multicolumn{5}{|c|}{ Learnable $\mathrm{S}-\mathrm{O}$ positive feedback > learnable $\mathrm{S}-\mathrm{O}$ negative feedback } \\
\hline Superior parietal & 0.007 & 100 & $5.65 / 3.94$ & $33,-54,60$ \\
\hline
\end{tabular}

hippocampal activity, whereas feedback processing for unlearnable associations involved relatively more ACC activity. Although we found that stimulus processing for unlearnable S-O associations involved more activity in occipital and inferior frontal gyrus regions, we did not find significant differences in our regions of interest for stimulus processing (hippocampus, striatum, midbrain, or ACC). The overall results support our hypothesis that learnability is one factor that affects memory system activations in association learning, and they suggest that the effects are primarily manifest at the time of feedback processing.

The hippocampus and ACC are both critical regions for learning and memory. In fMRI, hippocampal activity is implicated in declarative memory and association learning (e.g., Davachi 2006), whereas ACC activity is implicated in error and conflict monitoring of stimulus-response outcomes (e.g., Holroyd and Coles 2002; Rushworth et al. 2004, 2007; Critchley et al. 2005; Sarter et al. 2006). In this sense, the ACC is not thought of as a memory system itself that represents particular associations, but rather it is thought of as performing evaluation and/or control functions that are an integral part of learning. Thus, a plausible interpretation of our results is that processing feedback for learnable $\mathrm{S}-\mathrm{O}$ associations recruits relatively more declarative memory, and processing feedback for unlearnable $\mathrm{S}-\mathrm{O}$ associations recruits relatively more outcome monitoring. Given the problems with interpreting hippocampal activity relative to baseline (Stark and Squire 2001), however, it is not clear if hippocampal activity is suppressed for unlearnable $\mathrm{S}-\mathrm{O}$ associations, or if hippocampal activity is first suppressed overall and then recruited for learnable $\mathrm{S}-\mathrm{O}$ associations. A similar concern holds for ACC differences.

Midbrain activity was also found to be modulated by positive prediction errors for outcomes after both learnable and unlearnable stimuli. Dopaminergic projections from the midbrain are involved in the promotion of episodic learning in the hippocampus (Lisman and Otmakhova 2001; Schott et al. 2004; Lisman and Grace 2005; Wittman et al. 2005; Adcock et al. 2006), and they provide a reinforcement signal for stimulus-response learning in the basal ganglia (Schultz 2002; Montague et al. 2004). Thus, the presence of midbrain activity in fMRI does not necessarily distinguish memory systems or learning strategies.

We did not find significant differences in striatal regions based on either learnability or valence, as one might expect from previous studies of reward learning. However, there are several reasons why this might be the case, as discussed further below. First, given that midbrain activation is modulated by positive prediction errors for learnable and unlearnable $\mathrm{S}-\mathrm{O}$ associations, it may be that dopaminergic targets are also not differentially activated by learnability. Second, many learning studies use financial gains that may produce stronger effects, and/or less heterogeneous effects, than informational feedback (Seymour et al. 2007). In other words, the reward value of positive or negative informational feedback may be more ambiguous and/or subtle than primary rewards. Thus, depending on the task design, valence differences of informational feedback may be less consequential, or consequential in a different manner.

In many respects, the results here are consistent with related $\mathrm{S}-\mathrm{O}$ studies, which often show activation in MTL and ACC regions. However, the study here shows some differences in activation patterns that are worth pointing out. In one study that included random S-Os with two outcome choices, there was more hippocampal activation for positive feedback than negative feedback (Seger and Cincotta 2005). However, that study did not separate feedback processing from stimulus processing. The data here suggest that separating the stages of a trial may be critical in differentiating memory systems.

In a S-O learning task that did separate feedback and stimulus processing, Delgado et al. (2005) found that for random versus certain associations there was increased activation in caudate (Talairach $-8,3,4$ ) during feedback processing, decreased activation in ACC (Talairach $-9,42,23)$ during a long delay period $(10.5$ sec window), but no MTL differences. A major difference in the experiment is that Delgado et al. (2005) used financial rewards,
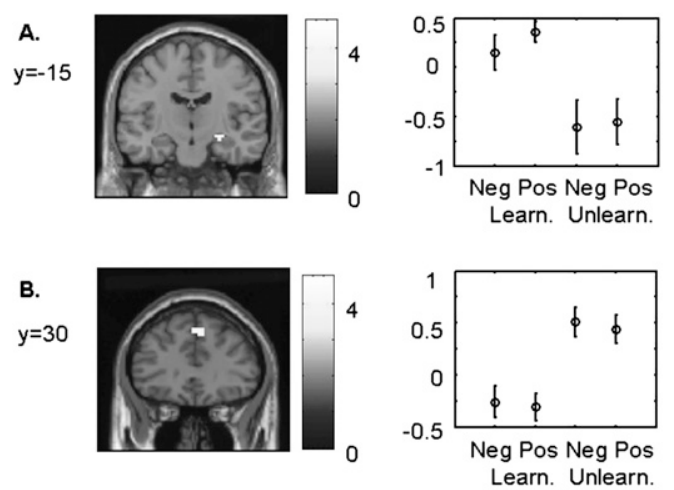

c.

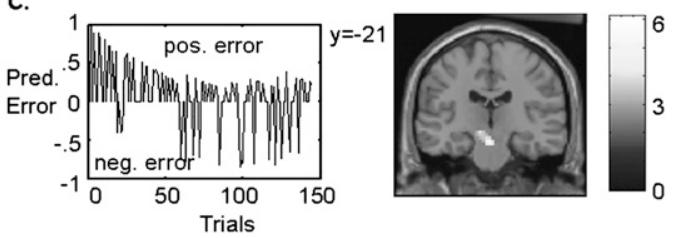

Figure 2. Activation and effect sizes are shown for feedback processing (A) Hippocampus cluster of increased activation at feedback processing for learnable - unlearnable $\mathrm{S}-\mathrm{O}$ associations. The peak of the cluster is at MNI coordinate $27,-15,-12$ (see Table 3). (B) ACC cluster of increased activation at feedback processing of unlearnable - learnable $\mathrm{S}-\mathrm{O}$ associations. The peak of the cluster is at MNI coordinate 6, 33, 45 (see Table 3). The graphs on the right side of $A$ and $B$ show the average effect size across subjects (with SEM) at the peak voxel corresponding to the figure on the left. (C, left) A sample of prediction errors for learnable stimuli from the model for one subject. Early in training positive feedback is unexpected and leads to higher positive prediction errors. Later in training, when positive feedback is expected, there are higher values for negative prediction errors. (Right) Positive prediction errors modulate activity in midbrain region at feedback processing for learnable $\mathrm{S}-\mathrm{O}$ associations. Peak voxels are at MNI $0,-21,-18$ and $-9,-24,-9$ (see Table 4). Color figures are available in Supplemental Material. 
Table 4. Contrast of effects size for parametric modulation by prediction errors

\begin{tabular}{|c|c|c|c|c|}
\hline & $P$-value & $\begin{array}{l}\text { Cluster } \\
\text { size }\end{array}$ & $\begin{array}{l}t \text {-Value/ } \\
Z \text {-score }\end{array}$ & $\begin{array}{c}\text { MNI } \\
\text { coordinates }\end{array}$ \\
\hline \multicolumn{5}{|c|}{ Positive prediction error for learnable-stimuli feedback $>$ baseline } \\
\hline Inferior frontal gyrus & 0.002 & 123 & $6.00 / 4.08$ & $30,36,15$ \\
\hline Left cerebellum & 0.001 & 161 & $5.83 / 4.02$ & $-21,-57,-36$ \\
\hline Right cerebellum & 0.026 & 81 & $4.48 / 3.42$ & $36,-57,-27$ \\
\hline \multirow[t]{2}{*}{ Midbrain } & 0.003 (SVC) & 41 & $6.07 / 4.11$ & $0,-21,-18$ \\
\hline & & & $4.74 / 3.56$ & $-9,-24,-9$ \\
\hline \multicolumn{5}{|c|}{ Positive prediction error for unlearnable-stimuli feedback $>$ baseline } \\
\hline Parietal & 0.009 & 89 & $5.18 / 3.75$ & $36,-54,63$ \\
\hline Midbrain & 0.060 (SVC) & 9 & $4.04 / 3.20$ & $-9,-21,-12$ \\
\hline
\end{tabular}

which could very well produce different activation patterns over the same dopaminergic target regions compared with the task used here.

In a previous study of weather prediction, Aron et al. (2004) reported ACC (Montreal Neurological Institute [MNI] 0, 30, 48) activity for negative - positive feedback. However, in probabilistic association learning, the occurrence of negative/positive feedback is correlated with low/high levels of learnability. Although we did not find ACC differences due to valence, we did find differences due to learnability with a similar peak location (MNI 6, 33, 45). Thus, the data here suggest that it is the uncertainty of outcomes, rather than the valence per se, which is more central to ACC activation (c.f. Botvinick et al. 2004; Rushworth et al. 2004). In fact, this is compatible with the hypothesis that uncertainty, not expected value of reward, is a primary factor that determines which memory system controls behavior (Daw et al. 2005; Behrens et al. 2007).

In our prior modeling work with the weather prediction task, we found striatal activation that increased with larger negative prediction error (Rodriguez et al. 2006), whereas here we find increased activation in midbrain for larger positive prediction errors. These differences could reflect differences in the task set-up that engender alternative learning strategies and/or variations in the way that positive/negative feedback is attended to. In particular, here we used four stimuli presented individually, two with high S-O conditional probabilities, whereas prior work used four stimuli presented individually or configurally for 14 possible unique combinations, with a variety of conditional probabilities. Also, having three outcomes, as in this study, versus two outcomes, as in the previous study, means that negative feedback is not as informative, especially early in learning. More generally, one could say that for both studies, the prediction error is related to learning or surprise in fMRI activity.

Also, in previous weather prediction studies, there was an overall decrease in MTL activity relative to baseline (Poldrack et al. 2001; Aron et al. 2004). In the data here, there was likewise a decrease in hippocampal activity at the feedback part of the trial for unlearnable stimuli. But there was also an increase in hippocampal activity during stimulus presentation, which possibly reflects the use of complex unfamiliar visual stimuli. This could help explain the recent finding that the hippocampus is equally active for feedback and observational learning in category learning (Cincotta and Seger 2007), task differences notwithstanding. The key is that it is not just the presentation of feedback or paired observations that separates memory system involvement, but whether or not the stimulus and $\mathrm{S}-\mathrm{O}$ associations themselves recruit the hippocampus at the time of stimulus or feedback processing.

It is worth pointing out that activation differences during stimulus processing (Table 1) could reflect the longer response time of unlearnable S-O associations. Differences during feedback processing, on the other hand, are not likely contaminated by motor response because the average response time was typically several seconds before feedback presentation. It is possible, however, that some differences in activity during feedback period are due to differences in anticipation of error that occur during the delay period leading up to the outcome presentation. There is, in fact, evidence that activity in the midbrain and ACC reflect uncertainty in anticipation of error (Fiorillo et al. 2003; Aron et al. 2004; Brown and Braver 2005). Future work could tease this apart better with longer fixed delays that allow analysis of the full time window after stimulus presentation.

In conclusion we have found evidence that learnability is one factor that affects memory systems in probabilistic $\mathrm{S}-\mathrm{O}$ association learning. Learnability here refers to the predictability of the $\mathrm{S}-\mathrm{O}$ associations, and the particular effect proposed is that declarative memory is differentially recruited, possibly as a function of outcome monitoring via the anterior cingulate. In this sense, the differential activity in ACC and hippocampus is functionally related to the acquisition and feedback processing for specific $\mathrm{S}-\mathrm{O}$ contingencies. An alternative interpretation, not mutually exclusive, is that the differential effects of learnability are related to meta-knowledge acquired about the stimuli. For example, subjects might decide to ignore feedback and simply make random guesses for the unlearnable $\mathrm{S}-\mathrm{O}$ associations, and/or simply apply an optimal rule for the learnable $\mathrm{S}-\mathrm{O}$ associations. If that were the case then the relative increase in ACC activity and decrease in hippocampal activity for unlearnable $\mathrm{S}-\mathrm{O}$ associations would be related to a suppression or absence of effort, lack of attention, and/ or rule-following, as opposed to some aspect of monitoring outcomes. However, we find that subjects generally continue to monitor response-outcome pairs and change predictions throughout the experiment, consistent with an interpretation that the differential activity is related to processing S-O contingencies. Perhaps with more subjects, one could disentangle learnability effects further by examining, for example, learnability only within subjects who perform probability matching or optimal decision making.

In addition to aspects of learnability, there are other questions raised about the patterns of brain activity we found. In particular, memory systems were not completely dissociated by learnability because we found midbrain activity modulated by positive prediction errors for both learnable and unlearnable associations, and we did not find significant differences in the striatum. Future studies could explore this further by testing the interaction of learnability with task conditions that dissociate the striatum and hippocampus, such as a feedback-based learning versus paired-observation learning paradigms (c.f. Poldrack et al. 2001).

\section{Materials and Methods}

\section{Task description}

Each trial consisted of a separate stimulus and outcome phase. The stimulus consisted of one of four possible complex visual figures along with the words "sun?," "snow?," or "rain?." The stimuli and words were presented for $2.5 \mathrm{sec}$, after which a fixation cross appeared for a jittered delay time, where the delay time was exponential distributed with a mean of 2.5 and a range of 2-6 sec (OptSeq software http://surfer.nmr.mgh.harvard.edu/optseq) (Dale 1999) 
Subjects were instructed to select one of three buttons with their right hand that correspond to the predicted outcomes as soon as possible and as accurately as possible. After the delay, the outcome was displayed on the screen in the same location as the three words in the stimulus phase (see Fig. 3A). The assignment of button press to outcome was always in the left-to-right order that matched the presentation on the screen.

Subjects were told to learn to predict the weather and that outcomes would be probabilistically associated with individual stimuli. The subjects were presented with a total of 144 trials, 36 for each stimulus. The trials were broken up into four runs, giving 36 trials per run and nine trials per stimulus in each run. A sample trial is given in Figure 3B. Subjects were shown the four stimuli together prior to scanning and presented with two sample trials. The assignment of S-Os and the outcome positions on the screen were randomized across subjects.

The probabilistic S-O assignments were made so that for two stimuli there was a high probability of being followed by one of the outcomes ("learnable" stimuli), and for the two other stimuli ("unlearnable" stimuli) there was pseudorandom assignment to any outcome. In fact, the number of outcomes that matched subject predictions (i.e., positive feedback) for the unlearnable stimuli was fixed by the experimental program so that subjects would be at chance performance across both stimuli. The program essentially kept a running average of performance and randomly reassigned outcomes during training. Using a running average has an advantage over truly random associations because it ensures that subjects do not accidentally experience a long run of positive feedback.

The S-O assignments are presented in Table 4. Notice that for learnable stimuli the probabilities were changed slightly in the last two runs in order to provide more trials of unexpected outcomes (i.e., negative feedback for predictable outcomes). The program returned more positive feedback for unlearnable stimuli in the last run also to provide more trials of unexpected outcomes (i.e., positive feedback for unpredictable outcomes). Although the change in percentage of matching outcomes in the last run is high for unlearnable stimuli (from 33\%-66\% positive feedback), over the whole experiment the change is relatively small (from 33\%$42 \%)$. This nonstationarity does not affect the main results that follow below, meaning that if we drop the last run from the analysis the contrasts that are significant (or marginal) stay significant (or marginal).

\section{Models}

We implemented a version of the Rescorla-Wagner model based a category learning application (see Gluck and Bower 1988). The model consisted of four input nodes, one for each stimulus, each with weighted connections to three output nodes, one for each possible weather outcome. On each trial, the activity for each output node was computed as:

A.

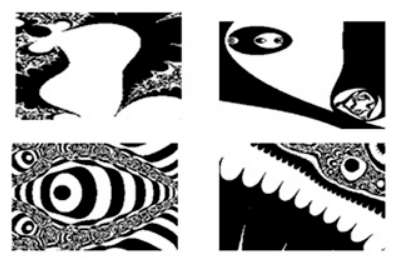

B.

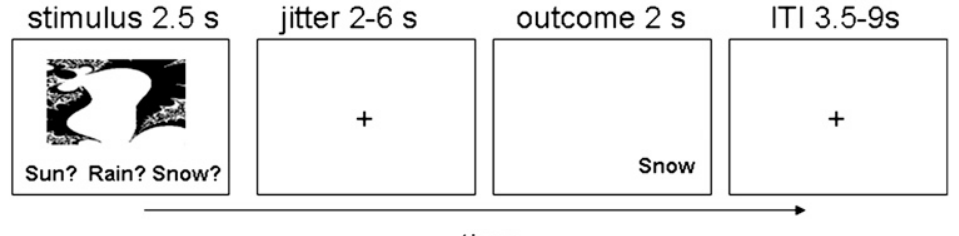

time

Figure 3. Sample stimuli $(A)$ and time line for a sample trial $(B)$.

$$
O_{i}=V_{i j} I_{j}
$$

and the weight change for that trial computed as:

$$
\Delta V_{i j}=\varepsilon\left(\lambda_{i}-O_{i}\right) I_{j},
$$

where $i=1,2$, or 3 indexes the output node, $j=1 \ldots 4$ indexes the input node, $O_{\mathrm{i}}$ is the $i$ th output node, $V_{\mathrm{ij}}$ is an association weight to $i$ from $j, I_{i}=1$ or 0 is the $i$ th input node, $\varepsilon$ is the learning rate, and $\lambda_{i}=1$ or 0 is the target value for the $i$ th output node. On each trial, the maximum value outcome was selected by the model as the outcome to predict (except the first trial where the outcome was randomly selected because initial weights were set to zero).

The prediction error for each trial is taken from the singular term $\left(\lambda_{i}-O_{i}\right)$ where $O_{i}$ is the maximum output on that trial. The target values, $\lambda_{i}$, were based on actual outcomes presented in each subject's experiment. We tried other estimates of prediction error, such as using target vectors based on the actual predictions made by each subject, or using the mean squared error from all output nodes. Neither of these worked as well in the fMRI analysis.

A reasonable value for the learning rate parameter was determined by comparing the percentage of correctly matching outcomes (i.e., positive feedback) for the model with the subject's percentage of correctly matching outcomes. A range of $\varepsilon=0.10$ 0.20 accounts equally well (within $1 \%$ ) for subject data, with an average of $69 \%$ overlap with subject predictions $(85 \%$ for learnable stimuli, $54 \%$ for unlearnable stimuli). Note that even though correctness is at chance for the unlearnable stimuli, neither the model nor subjects are making completely random choices. Rather, predictions are based in part on the short-term history of observed feedback. Related studies have used learning rate values in the range of $0.10-0.30$, (Glascher and Buchel 2006; Yacubian et al. 2006; Seymour et al. 2007). As done in other studies (Glascher and Buchel 2006), we choose a parameter value of 0.10 because it should presumably better capture the slower learning process and be less sensitive to fluctuations due to artifacts.

The model is designed to capture information about S-O association strengths, which is then used to as a proxy of subjects' predictions and expectations. Though subjects' predictions are likely a function of more than just S-O contingencies they may also depend on risk seeking and decision strategies. Future work could try to incorporate these other aspects into fMRI analysis, although it would require more parameters (e.g., Yechiam and Busemeyer 2005).

\section{FMRI acquisition and data analysis}

Fourteen subjects (ages 21-37, eight females) volunteered for this study. A Philips Eclipse 1.5T MR scanner (Philips Medical Systems) was used to acquire both T1-weighted anatomical volume images $\left(256 \times 256\right.$ matrix, $1-\mathrm{mm}^{3}$ voxels, spoiled gradient recalled acquisition in a steady state sequence) and T2*-weighted echo planar images (EPI; field of view of $25 \mathrm{~cm}, 64 \times 92$ matrix, $2.6 \times 3.9 \mathrm{~mm}$ pixels, TE $40 \mathrm{msec}$ ) with blood oxygenation level-dependent (BOLD) contrast. Each EPI volume comprised 22 4-mmthick axial slices separated by 1-mm gap, a TR (repetition time) of 2 sec. Volume slices were acquired in a descending interleaved order. The first four volumes were discarded to allow tissue magnetization to achieve a steady state.

Preprocessing and statistical analysis was performed with SPM2 (Wellcome Department of Neuroimaging, University College London). The data were slice time corrected, realigned across all scanning runs to the first volume of the first run, normalized to an EPI template in MNI space (Montreal Neurological 
Table 5. Probabilistic associations for learnable and unlearnable stimuli

\begin{tabular}{|c|c|c|c|c|}
\hline \multicolumn{5}{|c|}{ Learnable stimuli } \\
\hline & Scan run & Sun & Snow & Rain \\
\hline \multirow[t]{4}{*}{ Stimulus Figure 1} & 1 & $8 / 9$ & $1 / 9$ & 0 \\
\hline & 2 & $8 / 9$ & $1 / 9$ & 0 \\
\hline & 3 & $7 / 9$ & $2 / 9$ & 0 \\
\hline & 4 & $7 / 9$ & $2 / 9$ & 0 \\
\hline \multirow[t]{4}{*}{ Stimulus Figure 2} & 1 & 0 & $1 / 9$ & $8 / 9$ \\
\hline & 2 & 0 & $1 / 9$ & $8 / 9$ \\
\hline & 3 & 0 & $2 / 9$ & $7 / 9$ \\
\hline & 4 & 0 & $2 / 9$ & $7 / 9$ \\
\hline \multicolumn{5}{|c|}{ Unlearnable stimuli } \\
\hline & \multicolumn{2}{|c|}{ Scan run } & $\begin{array}{l}\text { Percentage correct } \\
\text { (fixed by program) }\end{array}$ & \\
\hline \multirow[t]{4}{*}{ Stimulus Figure 3} & \multicolumn{2}{|c|}{1} & $2 / 9$ & \\
\hline & \multicolumn{2}{|c|}{2} & $2 / 9$ & \\
\hline & \multicolumn{2}{|c|}{3} & $2 / 9$ & \\
\hline & \multicolumn{2}{|c|}{4} & $6 / 9$ & \\
\hline \multirow[t]{4}{*}{ Stimulus Figure 4} & \multicolumn{2}{|c|}{1} & $4 / 9$ & \\
\hline & \multicolumn{2}{|c|}{2} & $4 / 9$ & \\
\hline & \multicolumn{2}{|c|}{3} & $4 / 9$ & \\
\hline & \multicolumn{2}{|c|}{4} & $6 / 9$ & \\
\hline
\end{tabular}

stimuli) and $2 \times 2$ design for feedback (i.e., using regressors 3-6 above the $2 \times 2$ conditions are as follows: [1] optimal choice, learnable $\mathrm{S}-\mathrm{O}$ and [2] unlearnable $\mathrm{S}-\mathrm{O}$ versus [1] positive and [2] negative feedback). The primary comparisons of interest are between the learnability conditions.

Also, contrasts were applied to the parametric modulation regressors for prediction error. We planned to test parametric modulations for differences between learnability conditions as well as effects of modulation significantly different than zero.

Multiple comparisons across the whole brain or within regions of interest are corrected by Gaussian random field theory with a display threshold of $P<0.005$ and extent threshold of five voxels (Worsley et al. 1995). Based on prior hypothesis, four ROI have been selected to apply small volume correction (SVC). The regions were defined using WFU Pickatlas software (Maldjian et al. 2003) as follows, left and right hippocampus, left and right ventral striatum (12-mmradius sphere centered at $\pm 12,10,0)$, left and right midbrain area $(10 \mathrm{~mm}$ radius sphere centered at $\pm 8,-20,-18)$, anterior cingulate area (20-mm-radius sphere centered at coordinate $0,24,35$, intersected

Institute), smoothed with an 8-mm Gaussian kernel in 3D, and resliced to isotropic $3 \times 3 \times 3 \mathrm{~mm}$ voxels.

The general linear model was applied to individual subject data with separate regressors for stimulus and feedback presentations. The stimulus conditions were either learnable or unlearnable stimuli. The feedback presentations were separated according to positive or negative feedback, where positive (negative) refers to an outcome that matched (not matched) the prediction. The feedback for learnable stimuli was further separated according to whether the prediction was optimal or nonoptimal, where optimal refers to choosing the most likely outcome for a given stimulus. The unlearnable stimuli, of course, have no optimal choice and so feedback was not separated further. Thus, there were eight separate regressors related to stimuli and feedback: (1) learnable stimuli; (2) unlearnable stimuli; (3) positive feedback for learnable stimuli, optimal choice; (4) negative feedback for learnable stimuli, optimal choice; (5) positive feedback for unlearnable stimuli; (6) negative feedback for unlearnable stimuli; (7) positive feedback for learnable stimuli, nonoptimal choice; and (8) negative feedback for learnable stimuli, nonoptimal choice. Note that the number of trials for the last two regressors was highly variable among subjects. In fact, only five subjects had any trials for the seventh regressor, and six subjects had less than six trials of the eighth regressor. This low number of trials cautions against using those regressors in comparisons of interest. On the other hand, there was a range of nine to 12 trials for the fourth regressor among all subjects.

In addition to the above regressors, there were six covariates of no interest for estimated motion parameters and a constant regressor for each scanning run. As implemented in Statistical Parametric Mapping (SPM), a constant session-specific regressor accounts for baseline effects, so that a positive contrast of taskrelated regressors is implicitly a comparison of task effects - baseline. We also separately modeled missed trials in which no button response was recorded, which consisted of an extra stimulus and outcome regressor for each missed trial. Three subjects missed one trial, and three missed more than one (four, two, and two trials). Positive and negative prediction errors from the mathematical model were entered as parametric modulations of positive and negative feedback.

Contrasts of interest at the individual subject level were entered into $t$-tests in a second-level random effects group analysis, treating subjects as the random effects. In summary, the experiment is a $1 \times 2$ design for stimuli (learnable/unlearnable with gray matter of superior frontal gyrus, middle frontal gyrus, cingulate gyrus, and premotor regions). The striatal region was chosen based on our previous study that found activity modulated by prediction errors (Rodriguez et al. 2006), and was placed at the dorsal/ventral border so that it would include portions of caudate and putamen. The midbrain region was chosen because of its importance in reward prediction error studies (Schultz 2002), and the focus was guided by the atlas of Lucerna and colleagues (2002). The anterior cingulate was chosen because of the evidence that it is involved in error/conflict monitoring (e.g., Carter et al. 1998) and its hypothesized relevance to learning (e.g., Holroyd and Coles 2002). The focus was selected according to the surveys by Picard and Strick (2001) and Rushworth and colleagues (2004).

\section{Acknowledgments}

This work was supported by the Research Imaging Center at University of California, Irvine, and by a faculty development award from University of California, Irvine.

\section{References}

Abler, B., Walter, H., Erk, S., Kammerer, H., and Spitzer, M. 2006. Prediction error as a linear function of reward probability is coded in human nucleus accumbens. Neuroimage 31: 790-795.

Adcock, R.A., Thangavel, A., Whitfield-Gabrieli, S., Knutson, B., and Gabrieli, J.D. 2006. Reward-motivated learning: Mesolimbic activation precedes memory formation. Neuron 50: 507-517.

Aron, A.R., Shohamy, D., Clark, J., Myers, C., Gluck, M.A., and Poldrack, R.A. 2004. Human midbrain sensitivity to cognitive feedback and uncertainty during classification learning. J. Neurophysiol. 92: 1144-1152.

Behrens, T.E., Woolrich, M.W., Walton, M.E., and Rushworth, M.F. 2007. Learning the value of information in an uncertain world. Nat. Neurosci. 10: $1214-1221$.

Beninger, R.J., Wasserman, J., Zanibbi, K., Charbonneau, D., Mangels, J., and Beninger, B.V. 2003. Typical and atypical antipsychotic medications differentially affect two nondeclarative memory tasks in schizophrenic patients: A double-dissociation. Schizophr. Res. 61: 281292 .

Berns, G.S., McClure, S.M., Pagnoni, G., and Montague, P.R. 2001. Predictability modulates human brain response to reward. J. Neurosci. 21: 2793-2798.

Binder, J.R., Bellgowan, P.S., Hammeke, T.A., Possing, E.T., and Frost, J.A. 2005. A comparison of two fMRI protocols for eliciting hippocampal activation. Epilepsia 46: 1061-1070. 
Botvinick, M.M., Cohen, J.D., and Carter, C.S. 2004. Conflict monitoring and anterior cingulate cortex: An update. Trends Cogn. Sci. 8: 539-546.

Brown, J.W. and Braver, T.S. 2005. Learned predictions of error likelihood in the anterior cingulate cortex. Science 307: 1118-1121.

Carter, C.S., Braver, T.S., Barch, D.M., Botvinick, M.M., Noll, D., and Cohen, J.D. 1998. Anterior cingulate cortex, error detection, and the online monitoring of performance. Science 280: 747-749.

Cincotta, C.M. and Seger, C.A. 2007. Dissociation between striatal regions while learning to categorize via feedback and via observation. J. Cogn. Neurosci. 19: 249-265.

Cohen, N.J. and Squire, L.R. 1980. Preserved learning and retention of pattern-analyzing skill in amnesia: Dissociation of knowing how and knowing that. Science 210: 207-210.

Critchley, H.D., Tang, J., Glaser, D., Butterworth, B., and Dolan, R.J. 2005. Anterior cingulate activity during error and autonomic response. Neuroimage 27: 885-895.

Dale, A.M. 1999. Optimal experimental design for event-related fMRI. Hum. Brain Mapp. 8: 109-114.

Davachi, L. 2006. Item, context and relational episodic encoding in humans. Curr. Opin. Neurobiol. 16: 693-700.

Daw, N.D., Niv, Y., and Dayan, P. 2005. Uncertainty-based competition between prefrontal and dorsolateral striatal systems for behavioral control. Nat. Neurosci. 8: 1704-1711.

Delgado, M.R., Miller, M.M., Inati, S., and Phelps, E.A. 2005. An fMRI study of reward-related probability learning. Neuroimage 24: 862-873.

Eichenbaum, H., Otto, T., and Cohen, N.J. 1994. Two component functions of the hippocampal memory system. Behav. Brain Sci. 17: 449-517.

Fletcher, P.C., Anderson, J.M., Shanks, D.R., Honey, R., Carpenter, T.A., Donovan, T., Papadakis, N., and Bullmore, E.T. 2001. Responses of human frontal cortex to surprising events are predicted by formal associative learning theory. Nat. Neurosci. 4: 1043-1048.

Fiorillo, C.D., Tobler, P.N., and Schultz, W. 2003. Discrete coding of reward probability and uncertainty by dopamine neurons. Science 299: 18981902.

Foerde, K., Knowlton, B.J., and Poldrack, R.A. 2006. Modulation of competing memory systems by distraction. Proc. Natl. Acad. Sci. 103: 11778-11783.

Gabrieli, J.D.E., Brewer, J.B., Desmond, J.E., and Glover, G.H. 1997. Separate neural bases of two fundamental memory processes in the human medial temporal lobe. Science 276: 264-266.

Glascher, J. and Buchel, C. 2006. Formal learning theory dissociates brain regions with different temporal integration. Neuron 47: 295-306.

Gluck, M.A. and Bower, G.H. 1988. Evaluating an adaptive network model of human learning. J. Mem. Lang. 27: 166-195.

Haruno, M., Kuroda, T., Doya, K., Toyama, K., Kimura, M., Samejima, K., Imamizu, H., and Kawato, M. 2004. A neural correlate of reward-based behavioral learning in caudate nucleus: A functional magnetic resonance imaging study of a stochastic decision task. J. Neurosci. 24: 1660-1665.

Holroyd, C.B. and Coles, M.G. 2002. The neural basis of human error processing: Reinforcement learning, dopamine, and the error-related negativity. Psychol. Rev. 109: 679-709.

Kerns, J.G., Cohen, J.D., MacDonald, A.W., Cho, R.Y., Stenger, V.A., and Carter, C.S. 2004. Anterior cingulate conflict monitoring and adjustments in control. Science 303: 1023-1026.

Kirwan, C.B. and Stark, C.E. 2004. Medial temporal lobe activation during encoding and retrieval of novel face-name pairs. Hippocampus 14: 919930.

Knowlton, B.J., Mangels, J.A., and Squire, L.R. 1996. A neostriatal habit learning system in humans. Science 273: 1399-1402.

Lisman, J.E. and Grace, A.A. 2005. The hippocampal-VTA loop: Controlling the entry of information into long-term memory. Neuron 46: 703-713.

Lisman, J.E. and Otmakhova, N.A. 2001. Storage, recall, and novelty detection of sequences by the hippocampus: Elaborating on the SOCRATIC model to account for normal and aberrant effects of dopamine. Hippocampus 11: 551-568.

Lucerna, S., Salpietro, F.M., Alafaci, C., and Tomasello, F. 2002. In vivo atlas of deep brain structures. Springer-Verlag, Berlin, Germany.

Maldjian, J.A., Laurienti, P.J., Kraft, R.A., and Burdette, J.H. 2003. An automated method for neuroanatomic and cytoarchitectonic atlasbased interrogation of fMRI data sets. Neuroimage 19: 1233-1239.

Montague, P.R., Hyman, S.E., and Cohen, J.D. 2004. Computational roles for dopamine in behavioural control. Nature 431: 760-767.
Moody, T.D., Bookheimer, S.Y., Vanek, Z., and Knowlton, B.J. 2004. An implicit learning task activates medial temporal lobe in patients with Parkinson's disease. Behav. Neurosci. 118: 438-442.

O’Doherty, J.P., Dayan, P., Friston, K., Critchley, H., and Dolan, R.J. 2003. Temporal difference models and reward-related learning in the human brain. Neuron 38: 329-337.

Packard, M.G. and Knowlton, B.J. 2002. Learning and memory functions of the Basal Ganglia. Ann. Rev. Neurosci. 25: 563-593.

Picard, N. and Strick, P.L. 2001. Imaging the premotor areas. Curr. Opin. Neurobiol. 11: 663-672.

Poldrack, R.A., Clark, J., Pare-Blagoev, J., Shohamy, D., Creso Moyano, J., and Myers, C. 2001. Interactive memory systems in the human brain. Nature 414: $546-550$.

Rescorla, R. and Wagner, A. 1972. A theory of Pavlovian conditioning: Variations in the effectiveness of reinforcement and nonreinforcement. In Classical conditioning II: Current research and theory (eds. A. Black and W. Prokasy), pp. 64-99. Appleton Century Crofts, New York.

Rodriguez, P.F., Aron, A.R., and Poldrack, R.A. 2006. Ventral-striatal/ nucleus-accumbens sensitivity to prediction errors during classification learning. Hum. Brain Mapp. 27: 306-313.

Rushworth, M.F., Walton, M.E., Kennerley, S.W., and Bannerman, D.M. 2004. Action sets and decisions in the medial frontal cortex. Trends Cogn. Sci. 8: 410-417.

Rushworth, M.F., Buckley, M.J., Behrens, T.E., Walton, M.E., and Bannerman, D.M. 2007. Functional organization of the medial frontal cortex. Curr. Opin. Neurobiol. 17: 220-227.

Sarter, M., Gehring, W.J., and Kozak, R. 2006. More attention must be paid: The neurobiology of attentional effort. Brain Res. Brain Res. Rev. 2: 145 160.

Schott, B.H., Sellner, D.B., Lauer, C.J., Habib, R., Frey, J.U., Guderian, S., Heinze, H.J., and Duzel, E. 2004. Activation of midbrain structures by associative novelty and the formation of explicit memory in humans. Learn. Mem. 1: 383-387.

Schultz, W. 2002. Getting formal with dopamine and reward. Neuron 36: 241-263.

Seger, C.A. and Cincotta, C.M. 2005. The roles of the caudate nucleus in human classification learning. J. Neurosci. 25: 2941-2951.

Seymour, B., Daw, N., Dayan, P., Singer, T., and Dolan, R.J. 2007. Differentia encoding of losses and gains in the human striatum. J. Neurosci. 27: $4826-4831$.

Shanks, D.R., Tunney, R.J., and McCarthy, J.D. 2002. A re-examination of probability matching and rational choice. J. Behav. Decis. Making 15: 233-250.

Shohamy, D., Myers, C.E., Grossman, S., Sage, J., Gluck, M.A., and Poldrack, R.A. 2004. Cortico striatal contributions to feedback based learning: Converging data from neuroimaging and neuropsychology. Brain 127: 851-859.

Squire, L.R. 1992. Memory and the hippocampus: A synthesis from findings with rats, monkeys, and humans. Psychol. Rev. 99: 195-231.

Stark, C.E. and Squire, L.R. 2001. When zero is not zero: The problem of ambiguous baseline conditions in fMRI. Proc. Natl. Acad. Sci. 98: 1276012766.

Volz, K.G., Schubotz, R.I., and von Cramon, D.Y. 2003. Predicting events of varying probability: Uncertainty investigated by fMRI. Neuroimage 19: 271-280.

White, N.M. and McDonald, R.J. 2002. Multiple parallel memory systems in the brain of the rat. Neurobiol. 77: 125-184.

Wittman, B.C., Schott, B.H., Guderian, S., Frey, J.U., Heinze, H.J., and Duzel, E. 2005. Reward-related fMRI activation of dopaminergic midbrain is associated with enhanced hippocampus-dependent long-term memory formation. Neuron 45: 459-467.

Worsley, K.J., Poline, J.B., Vandal, A.C., and Friston, K.J. 1995. Tests for distributed, nonfocal brain activation. Neuroimage 2: 183-194.

Yacubian, J., Glascher, J., Schroeder, K., Sommer, T., Braus, D.F., and Buchel, C. 2006. Dissociable systems for gain- and loss-related value predictions and errors of prediction in the human brain. J. Neurosci. 26: 9530-9537.

Yechiam, E. and Busemeyer, J.R. 2005. Comparison of basic assumptions embedded in learning models for experience-based decision making. Psychon. Bull. Rev. 12: 387-402.

Received August 15, 2008; accepted in revised form February 23, 2009. 


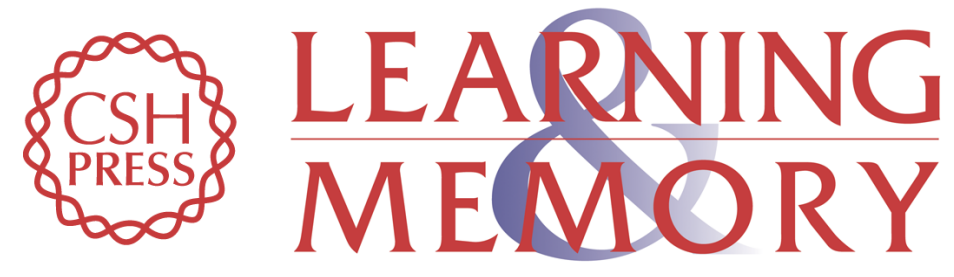

\section{Stimulus-outcome learnability differentially activates anterior cingulate and hippocampus at feedback processing}

Paul F. Rodriguez

Learn. Mem. 2009, 16:

Access the most recent version at doi:10.1101//m.1191609

Supplemental
Material http://learnmem.cshlp.org/content/suppl/2009/05/12/16.5.324.DC1

References This article cites 56 articles, 14 of which can be accessed free at: http://learnmem.cshlp.org/content/16/5/324.full.html\#ref-list-1

License

Email Alerting Receive free email alerts when new articles cite this article - sign up in the box at the Service top right corner of the article or click here. 